\title{
Modeling and Control of a Power-Split Hybrid Vehicle
}

\author{
Jinming Liu and Huei Peng
}

\begin{abstract}
Toyota Hybrid System (THS) is used in the current bestselling hybrid vehicle on the market-the Toyota Prius. This hybrid system contains a power-split planetary gear system which combines the benefits of series and parallel hybrid vehicles. In this paper, we developed a dynamic model of the THS powertrain and then apply it for model-based control development. Two control algorithms are introduced: one based on the stochastic dynamic programming method, and the other based on the equivalent consumption minimization strategy. Both approaches determine the engine power based on the overall vehicle efficiency and apply the electrical machines to optimize the engine operation. The performance of these two algorithms is assessed by comparing against the dynamic programming results, which are non-causal but provide theoretical benchmarks for other implementable control algorithms.
\end{abstract}

Index Terms-Dynamics, dynamic programming, hybrid vehicles, modeling, nonlinear systems, power control, power management, vehicle powertrain.

\section{INTRODUCTION}

$\mathbf{T}$ HE PURSUIT of improved fuel economy for ground vehicles accelerated in recent years due to the increased price of fossil fuel and the concerns of environmental impacts. Hybrid electric vehicles (HEVs) seem to be the most promising short-term solution and are under enthusiastic development by many automotive companies. An HEV adds an electric power path to the conventional powertrain, which helps to improve fuel economy by engine right-sizing, load leveling, and regenerative braking. A right-sized engine has better fuel efficiency and smaller heat loss. The reduced engine power is compensated by an electrical machine (or machines). Compared with internal combustion engines, electric machines provide torque more quickly, especially at low vehicle speed. Therefore, launching performance can be improved even with reduced overall rated power. Load leveling can also be achieved by adding the electrical path, which enables the engine to operate more efficiently, independent from the road load. Regenerative braking allows the electric machine to capture part of the vehicle kinetic energy and recharge the battery when the vehicle is decelerating.

\footnotetext{
Manuscript received January 4, 2007; revised June 26, 2007. Manuscript received in final form January 17, 2008. First published June 10, 2008; current version published October 22, 2008. Recommended by Associate Editor C. Bohn. This work was supported by the Automotive Research Center of the University of Michigan, a Center of Excellence sponsored by the U.S. Army TARDEC under Contract DAAE07-98-C-R-L008.

The authors are with the Department of Mechanical Engineering, University of Michigan, Ann Arbor, MI 48109-2133 USA (e-mail: jinmingl@umich.edu; hpeng@umich.edu).

Color versions of one or more of the figures in this paper are available online at http://ieeexplore.ieee.org.

Digital Object Identifier 10.1109/TCST.2008.919447
}

Based on the mechanical architecture, HEVs can be divided into three categories: parallel hybrids, series hybrids, and power-split hybrids. The parallel configuration, as shown in Fig. 1(A), includes two separate power paths: the mechanical path and the electrical path. Each power path can drive the vehicle individually or collaboratively. The main drawback of the parallel configuration is that a single electric machine is typically used both as a generator and as a motor. The electric power assistance must be constrained to avoid draining the battery and frequent role-reversal may be necessary. The series configuration, as shown in Fig. 1(B), only applies a motor (or motors) to drive the wheels. The motor power is supplied by either a battery, or a generator transforming the engine power into electrical power, or both. Since the engine operation is independent of the vehicle speed and road load, it can operate near its optimal condition almost all the time. A disadvantage of such configuration, however, is that the efficiency of the electric machine(s) will reduce the overall power-train efficiency [1].

The power-split hybrids combine the previous two configurations with a power-split device, as shown in Fig. 1(C). It is appealing because under proper control it can be designed to take advantage of both parallel and series types and avoid their disadvantages.

The power-split mechanisms were studied as early as the 1970s [2]. Earlier versions of such devices appeared in the hydrostatic power-split transmission commonly used on lawn tractors. Miller et al. [3] provided a historical perspective of the power-split device development. The Toyota Hybrid System (THS), the core of the first commercial power-split HEV offered in 1997 in Japan, the Toyota Prius, was described in [4]-[7]. The early model of the Prius was tested by the Argonne National Lab [8] and the experiment data were used for modeling the vehicle in PSAT [9] and ADVISOR [10]. A comparative study between the THS and another hybrid design, the Honda Insight, is done by Duoba et al. [11]. In 2004, Toyota released an improved THS system (THS II). Studies [12]-[14] showed that the main differences between the original and the new THS power-trains mainly are the component sizing instead of power-train architecture. The THS II power-split system was adopted and improved for higher-load vehicles as the Toyota Highlander and the Lexus RH400, as described by Hermance et al. [15].

Few papers have been published in the literature on the modeling and control of power-split hybrid vehicles. Rizoulis et al. [16] presented a mathematical model of a vehicle with a power-split device based on the steady-state transmission performance. A power-split hybrid vehicle model was developed by Zhang et al. [17] to study the optimal control algorithm. This dynamic model is simplified without detailed component behavior. Miller [18] summarized the models of the current devel- 


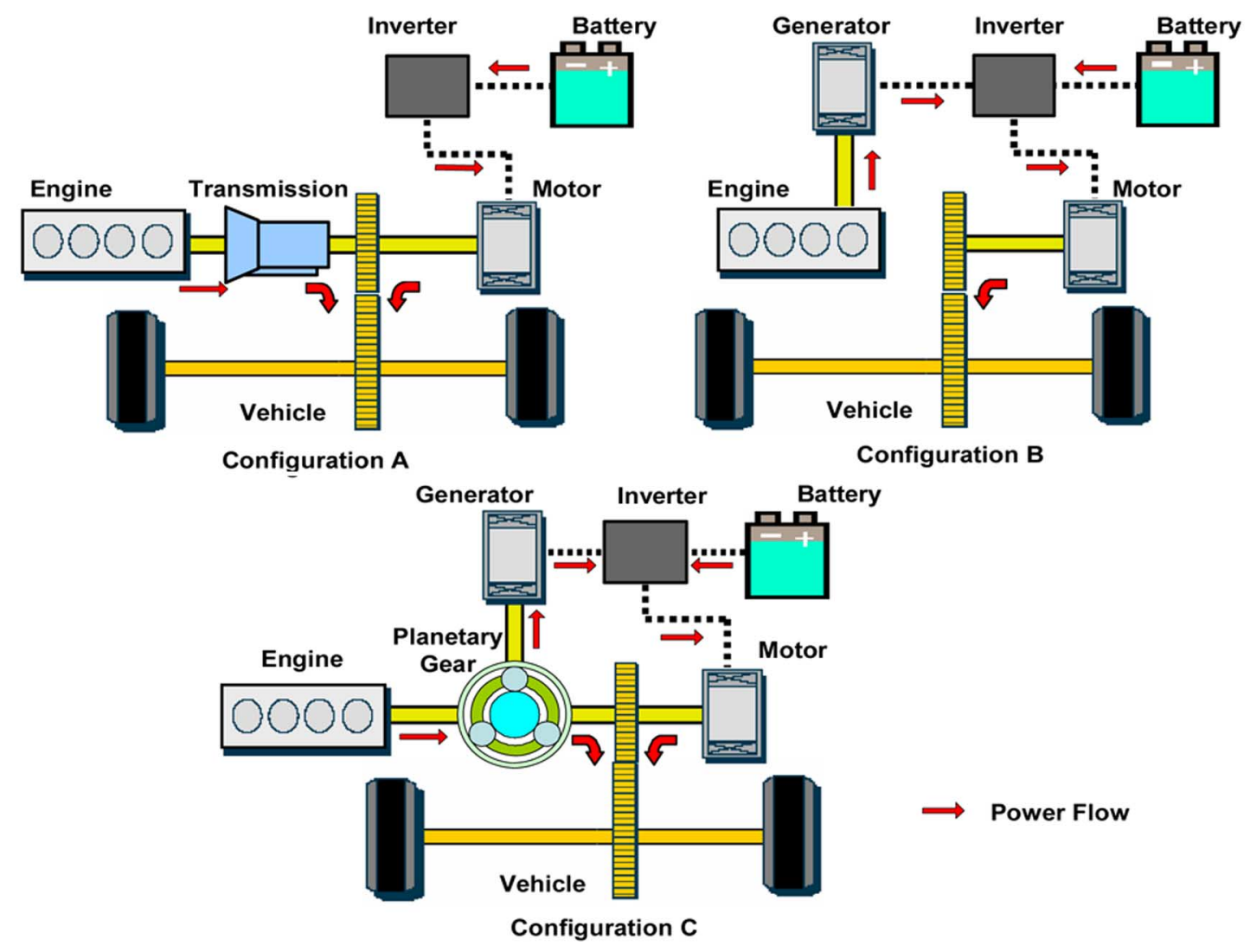

Fig. 1. Hybrid vehicle configurations: (A) parallel; (B) series; and (C) power-split (parallel/series).

oping power-split HEV architectures. The power-management control algorithm for the THS, or power-split hybrid systems in general were not analyzed in depth, except the few papers discussing rule-based algorithms for THS.

A three-state dynamic vehicle model is presented in this paper. It can be applied to describe either THS or THS II by properly choosing the component parameters. Using this dynamic vehicle model, two optimal control designs are introduced and analyzed. The first design is based on the stochastic dynamic programming (SDP) approach, introduced by Lin et al. [19]. In this approach, the vehicle model is deterministic but the driver power demand is stochastic, which reflects the fact that the optimization is not for any specified driving cycle, but rather for general driving conditions with known power demand probabilities. In this paper, the SDP approach is modified and applied to a power-split HEV. The second design studied in this paper is the equivalent consumption minimization strategy (ECMS) algorithm, which is proposed by Paganelli et al. [20]. This algorithm is based on an instantaneous optimization concept. The ECMS concept introduced in [20] was developed for a parallel HEV. This algorithm needs to be modified before it can be applied to power-split hybrids because the powertrain dynamics is completely different and there are two electrical machines rather than one. The modified ECMS for power-split hybrids is another contribution of this paper.

To assess the performance of the SDP and ECMS algorithms, the deterministic DP solutions for the driving cycles (EPA urban and highway cycles) are used as benchmarks for comparisons. The DP solutions are benchmarks rather than implemented control algorithms because they are non-causal in nature.

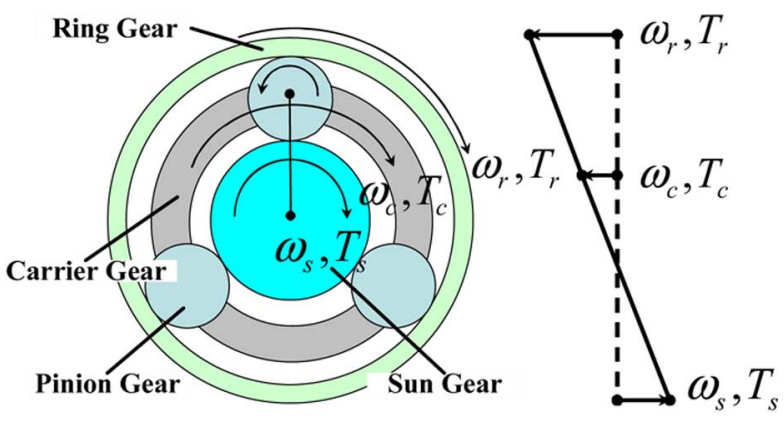

Fig. 2. Planetary gear and its level diagram analogy.

\section{Modeling OF THE THS}

THS uses a planetary gear as its power-split device [7]. As shown in the left-hand side of Fig. 2, a planetary gear consists of a ring gear, a sun gear, a carrier gear, and several pinion gears. Due to the mechanical connections through gear teeth, the rotational speeds of the ring gear $\omega_{r}$, sun gear $\omega_{s}$, and the carrier gear $\omega_{c}$ satisfy the following relationship:

$$
\omega_{s} S+\omega_{r} R=\omega_{c}(R+S)
$$

where $R$ and $S$ are the radii of the ring gear and sun gear, respectively. A lever diagram [21] is commonly used to describe this speed constraint, which simplifies the dynamic analysis for the planetary gear set, as shown in the diagram in the right-hand 


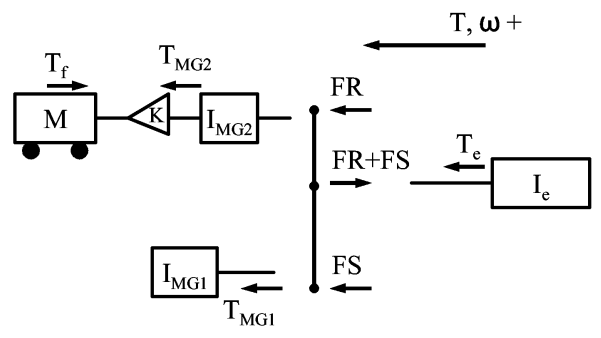

Fig. 3. Free-body diagram of the mechanical path of THS.

side of Fig. 2. The length of the arrow represents the magnitude of the rotational speed of each gear, while the direction reflects the sign.

Fig. 3 shows the free-body diagram of the mechanical path of the THS powertrain. The sun gear, carrier gear, and ring gear are connected to one motor/generator (MG1), engine, and vehicle, respectively. In addition, another electric motor/generator (MG2) is also attached to the ring gear, which enables direct motor propulsion and efficient regenerative braking. The power generated by the engine is split into two paths: a mechanical path and an electrical path. The mechanical path consists of power transferred from the carrier gear directly to the ring gear, which is connected to the vehicle drive axle. The electrical path takes the rest of the engine power and transforms it into electricity through the MG1. This power is then either charged into the battery or supplied to the MG2.

The dynamic equations were described in [22] and are summarized in the following. By assuming zero pinion gear inertia and neglect all vehicle dynamics except along the longitudinal direction, the governing equations are

$$
\begin{aligned}
\dot{\omega}_{\mathrm{MG} 1}\left(I_{\mathrm{MG} 1}+I_{s}\right)= & F \cdot S-T_{\mathrm{MG} 1} \\
\dot{\omega}_{e}\left(I_{e}+I_{c}\right)= & T_{e}-F \cdot R-F \cdot S \\
\dot{\omega}_{r}\left(\frac{R_{\mathrm{tire}}^{2}}{K} m+I_{\mathrm{MG} 2} K+I_{r} K\right)= & \left(T_{\mathrm{MG} 2}+F \cdot R\right) K-T_{f} \\
& -m g f_{r} R_{\mathrm{tire}} \\
& -0.5 \rho A C_{d}\left(\frac{\omega_{r}}{K}\right)^{2} R_{\mathrm{tire}}^{3}
\end{aligned}
$$

where $T_{\mathrm{MG} 1}, T_{\mathrm{MG} 2}$, and $T_{e}$ are the torques generated by the MG1, MG2, and engine, respectively; $I_{r}, I_{s}$, and $I_{c}$ are the inertias of the ring gear, sun gear, and carrier gear. $I_{\mathrm{MG} 1}, I_{\mathrm{MG} 2}$, and $I_{e}$, are the inertias of the power sources. $F$ represents the internal force on the pinion gears and $m$ is the vehicle mass. $T_{f}$ is the brake torque applied by the friction brake system. $K$ is the final drive ratio, $R_{\text {tire }}$ is the tire radius, $f_{r}$ is the rolling resistance coefficient, and $0.5 \rho A C_{d}$ represents the aerodynamic drag resistance. Equations (1)-(4) can be presented in the matrix form shown in (5) at the bottom of the page.

Although there are four equations, the number of mechanical degrees of freedom of this system is two. Engine speed $\omega_{e}$ and the ring gear speed $\omega_{r}$, which is proportional to the vehicle speed, are chosen as the two states for the mechanical motions. The fact that the engine speed is independent from the vehicle speed indicates this power-split device is a type of continuously variable transmission (CVT). The speed ratio, however, is manipulated through controlling the speed of MG1. Therefore, it is sometimes referred to as an electronic variable transmission (EVT) to differentiate it from other CVTs.

One additional state variable, the battery state of charge (SOC), needs to be defined to reflect battery energy status. It is calculated from the following equation:

$$
\mathrm{SO} C=-\frac{I_{\text {batt }}}{Q_{\max }}
$$

where $I_{\text {batt }}$ is the battery current and $Q_{\max }$ is the battery capacity. For simplicity, the internal resistance battery model is used, which results in

$$
P_{\mathrm{batt}}=V_{\mathrm{oc}} I_{\mathrm{batt}}-I_{\mathrm{batt}}^{2} R_{\mathrm{batt}}
$$

where $V_{\mathrm{oc}}$ is the battery open circuit voltage, $R_{\mathrm{batt}}$ is the battery resistance, and they both are functions of SOC. Note here when $P_{\text {batt }}$ is positive, the battery is discharging. When it is negative, the battery is charging. The battery power flows through the inverter to supply power to the electrical machines, and the relationship is governed by

$$
P_{\mathrm{batt}}=\left(T_{\mathrm{MG} 1} \omega_{\mathrm{MG} 1} \eta_{\mathrm{MG} 1}^{k} \eta_{i 1}^{k}+T_{\mathrm{MG} 2} \omega_{\mathrm{MG} 2} \eta_{\mathrm{MG} 2}^{k} \eta_{i 2}^{k}\right)
$$

where $\eta_{\mathrm{MG} 1}$ and $\eta_{\mathrm{MG} 2}$ are the efficiencies of the electric machines. $\eta_{i 1}$ and $\eta_{i 2}$ are the corresponding efficiencies of the inverters. The exponent $k=-1$ when the battery is discharged

$$
\begin{array}{r}
{\left[\begin{array}{cccc}
I_{s}+I_{\mathrm{MG} 1} & 0 & 0 & -S \\
0 & I_{c}+I_{e} & 0 & R+S \\
0 & 0 & \frac{R_{\text {tire }}^{2}}{K} m+I_{\mathrm{MG} 2} K+I_{r} K & -K R \\
S & -(R+S) & R & 0
\end{array}\right]\left[\begin{array}{c}
\dot{\omega}_{\mathrm{MG} 1} \\
\dot{\omega}_{e} \\
\dot{\omega}_{r} \\
F
\end{array}\right]} \\
\\
\quad=\left[\begin{array}{c}
T_{\mathrm{MG} 1} \\
T_{e} \\
K T_{\mathrm{MG} 2}-T_{\mathrm{fb}}-m g f_{r} R_{\text {tire }}-0.5 \rho A C_{d}\left(\frac{\omega_{r}}{K}\right)^{2} R_{\text {tire }}^{3} \\
0
\end{array}\right]
\end{array}
$$


TABLE I

MAIN PARAMETERS USED IN THE THS MODEL

\begin{tabular}{cc}
\hline \hline Parameters & Values \\
Vehicle mass & $1254 \mathrm{~kg}$ \\
Wheel radius & $0.287 \mathrm{~m}$ \\
Frontal area & $2.52 \mathrm{~m}^{2}$ \\
Air drag coefficient & 0.3 \\
Final drive ratio & 3.9 \\
Rolling resistance coefficient & 0.015 \\
Engine inertia & $0.18 \mathrm{~kg} \mathrm{~m}^{2}$ \\
MG1 inertia & $0.023 \mathrm{~kg} \mathrm{~m}^{2}$ \\
MG2 inertia & $0.023 \mathrm{~kg} \mathrm{~m}^{2}$ \\
\hline \hline
\end{tabular}
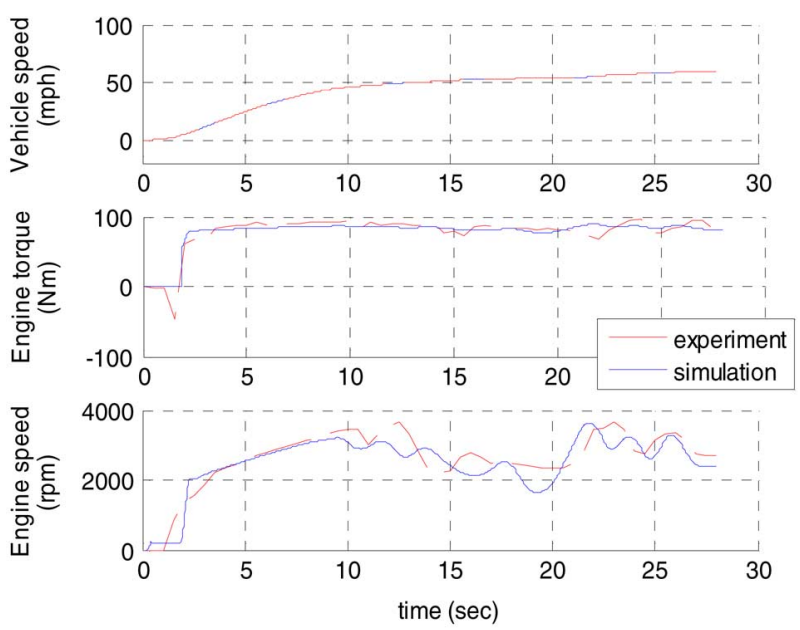

Fig. 4. Engine simulation results compared with published experiment results under the same driving cycle. (The experiment data is sampled at $2 \mathrm{~Hz}$ ).

and $k=1$ when it is charged. From (6) to (8), we have (9), shown at the bottom of the page.

This equation, together with the mechanical dynamics in (5), describes the dynamics of the THS vehicle.

For the purpose of model validation, this model is put under the command of a rule-based control strategy [7]. In the simulation, vehicle parameters, engine maps and efficiency torque, and fuel consumption tables are obtained from ADVISOR 2002 [10]. The key parameters are listed in Table I. Fig. 4 presents the comparison between the simulation results and the experiment results from [11]. Since we do not know the control gains precisely and considering all the simplifying assumptions we have made, the behavior of this model was found to agree with the actual system quite well. The rule-based control in this simulation was explained in detail in our earlier publication [22].

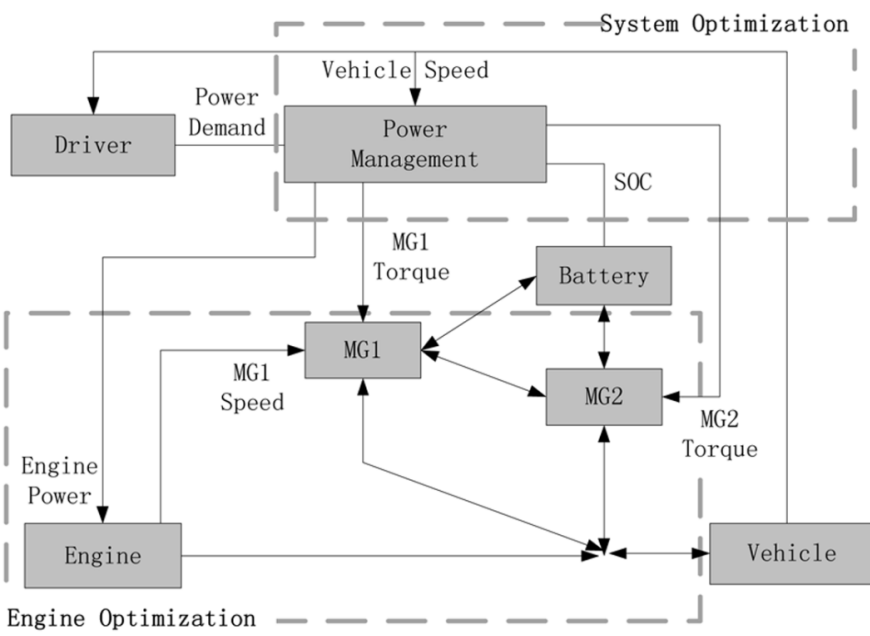

Fig. 5. Two-step control of the THS power train showing system optimization and engine optimization.

\section{POWER-SPlit AND ENGINE OPTIMIZATION}

In a power-split device, the engine speed can be decoupled from the vehicle load. Therefore, the engine can operate efficiently, similar to a series hybrid. This is beneficial for fuel economy during city driving, where many stop-and-go's occur. For highway driving, similar to a parallel hybrid, power flow is mostly through the mechanical path to improve fuel economy.

To fully realize the benefits of a power-split hybrid, the engine is controlled by manipulating the two electrical power machines. They both serve as either a motor or a generator, depending on the driving conditions. The MG in a speed control mode is controlled to manipulate the speed of the engine and the MG in a torque control mode helps to satisfy the torque requirement. In the THS, the MG1 is applied as the speeder and the MG2 is applied as the torquer [5]. To do the regenerative braking, MG2 is controlled to apply the braking torque up to its torque capacity and the friction brake fulfills the rest of the torque demand.

A divide-and-conquer architecture is suggested by Toyota in [5] to decouple the multiple input control design into two steps: system optimization and engine optimization (see Fig. 5). The system optimization specifies the engine power demand. Then the engine optimization controls the engine operation. The engine optimal controller selects a precalculated optimal engine speed based on the engine power command given by the system optimal controller. This desired speed is then achieved by manipulating the electric machine MG1 by using the speed relationship imposed by the lever diagram. Depending on the torque capacity and speed range of MG1, the desired engine speed may not be achievable, or even if it is, may be achievable after a transient. The power surplus or deficit (difference between desired

$$
\mathrm{SO} C=-V_{\mathrm{oc}}-\sqrt{\frac{V_{\mathrm{oc}}^{2}-4\left(T_{\mathrm{MG} 1 \omega \mathrm{MG} 1} \eta_{\mathrm{MG} 1}{ }^{k} \eta_{i 1}^{k}+T_{\mathrm{MG} 2{ }^{\omega} \mathrm{MG} 2} \eta_{\mathrm{MG} 2} \eta_{i 2}^{k}\right) R_{\mathrm{batt}}}{2 R_{\mathrm{batt}} Q_{\max }}}
$$




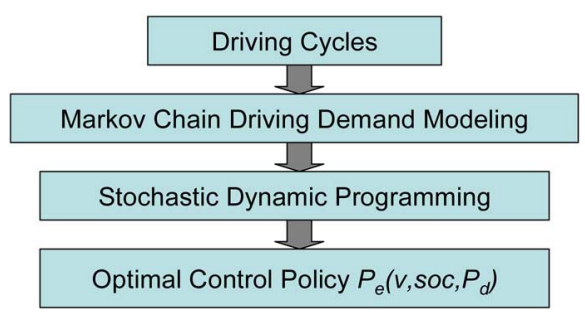

Fig. 6. SDP design process.

power and engine power) is then supplied by the other electric machine MG2. This procedure can also be found in [6].

The engine optimization process explained above is enginecentric. It maximizes engine efficiency for each required engine power level. However, the system optimization, the process of choosing a proper engine power level to increase the overall vehicle efficiency, has not been explained. This control decision should be comprehensive and should fulfill the driving demand, maintain proper battery SOC, and satisfy component imposed inequality constraints such as rotational speed and torque limit of the electrical machines. Moreover, it is desirable that the power management decision leads to certain optimality such as maximizing fuel economy. Rule-based control can provide guidance for improving fuel efficiency but cannot guarantee optimality in any rigorous sense.

In the following, two control algorithms are presented. The first is an infinite horizon optimal policy, which minimizes fuel consumption $E(t)$ over an infinite horizon

$$
\min \left(\int_{0}^{\infty} E(t) d t\right)
$$

The second approach is based on instantaneous optimization. The control action is determined based on minimization of instantaneous fuel consumption and the overall fuel consumption becomes

$$
\int(\min (E(t))) d t
$$

\section{Stochastic Dynamic Programming}

Lin et al. [19] proposed a SDP control approach for a parallel hybrid vehicle. As shown in Fig. 6, this approach extracts an optimal control policy from a Markov chain driver model, based on the power demand statistics of multiple driving cycles. This approach is modified and applied to the power-split hybrid vehicle as follows.

The objective of the SDP control algorithm is to find the optimal control policy $\pi$ that maps observed states SOC, vehicle speed $v$, and the instantaneous power demand $P_{d}$ to the control decision $P_{e}$, the engine power demand. Note only two deterministic states, SOC and $v$, are adopted here, which is different from the three deterministic states explained in the modeling section. This is to simplify the dimension of the model to reduce the computation cost for the SDP. To do so, the engine speed is

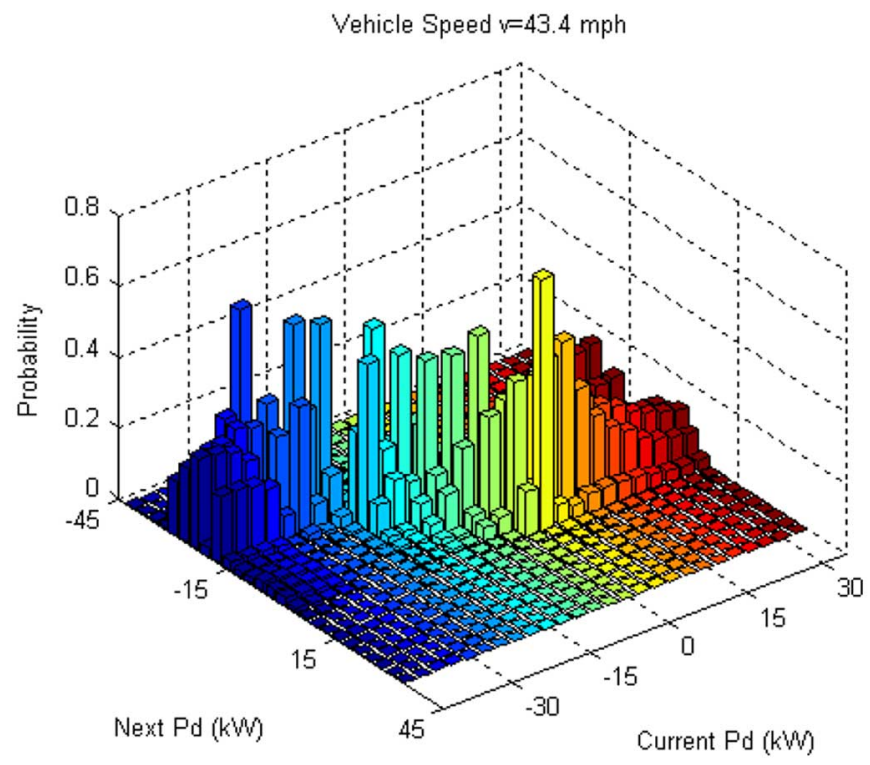

Fig. 7. Example of power demand probability map.

mapped into the current engine power demand by assuming that the engine operates on the predetermined curve, meaning, for each engine power, the optimal engine speed can be calculated. The third state, driving power demand $P_{d}$ is a random variable with known probability density function. Determining proper statistical characteristics of $P_{d}$ is not a science and depends on engineering judgment and available information (e.g., updated traffic and road condition ahead).

In this paper, the driving power demand is modeled to be generated from a stationary Markov chain. Four standard driving cycles, WVUCITY, WVUSUB, WVUINTER, and UDDSHDV (original data from ADVISOR 2002), were selected to represent mixed city, suburban, and highway driving conditions. From these driving cycles and vehicle parameters, the driving power $P_{d}$ can be calculated as a function of vehicle speed $v$. The observed pair $\left(P_{d}, v\right)$ is further mapped onto a sequence of quantized states $\left(P_{d}^{\prime}, v^{\prime}\right)$. The transition probability could then be estimated by the maximum-likelihood estimator, which counts the observation data as

$$
\hat{p}_{i l, j}=m_{i l, j} / m_{i l} \quad \text { if } m_{i l} \neq 0
$$

where $m_{i l, j}$ is the number of times the transition from $P_{d}^{i}$ to $P_{d}^{j}$ has occurred at vehicle speed state $v^{l}$ and $m_{i l}=\sum_{j=1}^{n} m_{i l, j}$ is the total event counts that $P_{d}^{i}$ has occurred at speed $v^{l}$. If the event count $m_{i l}$ is zero (e.g., due to inadequate richness of the driving cycles), the probabilities of those points are estimated by the information from the points around them. Fig. 7 shows an example probability map under a given speed.

Based on this stochastic Markov model, we formulated an infinite horizon SDP. The optimal control policy is extracted by minimizing the cost function $J_{\pi}$, the expected cost under control law $\pi$, over an infinite horizon

$$
J_{\pi}(x)=g(x, u)+\gamma \sum_{x^{\prime}} p_{x x^{\prime}} J_{\pi}\left(x^{\prime}\right)
$$


where

$$
\begin{aligned}
g(x, u,) & =\text { fuel }_{k}+\alpha \Delta_{\mathrm{SOC}}^{2} \\
\Delta_{\mathrm{SOC}} & = \begin{cases}\mathrm{SOC}_{k}-\mathrm{SOC}_{d} & \mathrm{SOC}_{k}<\mathrm{SOC}_{d} \\
0 & \mathrm{SOC}_{k}>=\mathrm{SOC}_{d}\end{cases}
\end{aligned}
$$

where the fuel consumption at each time step fuel ${ }_{k}$ is to be minimized and battery $\mathrm{SOC}_{k}$ is penalized with a penalty factor $\alpha$ when it is below the desired value $\mathrm{SOC}_{d} . J_{\pi}(x)$ indicates the resulting expected cost when the system starts at a given state and follows the policy $\pi$ thereafter. $0<\gamma<1$ is the discount factor. It is determined to weight the expected cost. $u$ is the control signal obtained from the control policy $\pi . x$ and $x^{\prime}$ are the current states and the next states. $p_{x x^{\prime}}$ is the transition probability between these two states. The optimization problem is subject to a set of inequality constraints arising from component speed, torque and SOC characteristics

$$
\begin{aligned}
\omega_{e_{-} \min } & \leq \omega_{e_{-} k+1} \leq \omega_{e_{-} \max } \\
\omega_{\mathrm{MG} 1_{-} \min } & \leq \omega_{\mathrm{MG} 1 \_k+1} \leq \omega_{\mathrm{MG} 1_{-} \max } \\
T_{\mathrm{MG} 1_{-} \min } & \leq T_{\mathrm{MG} 1_{-}+1} \leq T_{\mathrm{MG} 1_{-} \max } \\
\omega_{\mathrm{MG} 2_{-} \min } & \leq \omega_{\mathrm{MG} 2_{-k+1}} \leq \omega_{\mathrm{MG} 2_{-} \max } \\
T_{\mathrm{MG} 2_{-} \min } & \leq T_{\mathrm{MG} 2_{-}+1} \leq T_{\mathrm{MG} 2_{-} \max } \\
\mathrm{SOC}_{\min } & \leq \mathrm{SOC}_{k+1} \leq \mathrm{SOC}_{\max } .
\end{aligned}
$$

These inequality constraints are implemented by assigning large penalty to control decisions that violate these constraints.

The SDP problem is solved through a policy iteration algorithm, which consists of a policy evaluation step and a policy improvement step. This algorithm is solved iteratively until the cost function $J_{\pi}$ converges. In the policy evaluation step, given a desired power $P_{d}$, starting with an initial policy $\pi$, we calculate the corresponding cost function $J_{\pi}(x)$. Then a new policy is determined through the equation

$$
\pi\left(P_{d}\right)=\arg \min \left[g(x, u)+\gamma \sum_{x^{\prime}} p_{x x^{\prime}} J_{\pi}\left(x^{\prime}\right)\right] .
$$

After the new policy is obtained, we go back to the policy evaluation step to update the cost function by using the new policy. This process is repeated until $J_{\pi}$ converges within a selected tolerance level. The control policy generated is time-invariant and causal and has the form of nonlinear full-state feedback laws. An example map is shown in Fig. 8, notice that the accuracy is limited by the grid size on each state.

\section{ECMS WiTH KINEMATIC CONSTRAINS}

The ECMS is an instantaneous optimization algorithm introduced by Paganali [20]. The driving power demand $P_{d}$ is assumed to be always fulfilled by the engine power $P_{e}$ and the electrical machine power $P_{\text {elec }}$

$$
P_{d}=P_{e}+P_{\text {elec }} .
$$

When we are solving a power management problem for a hybrid vehicle, with the goal of minimizing fuel consumption, it is

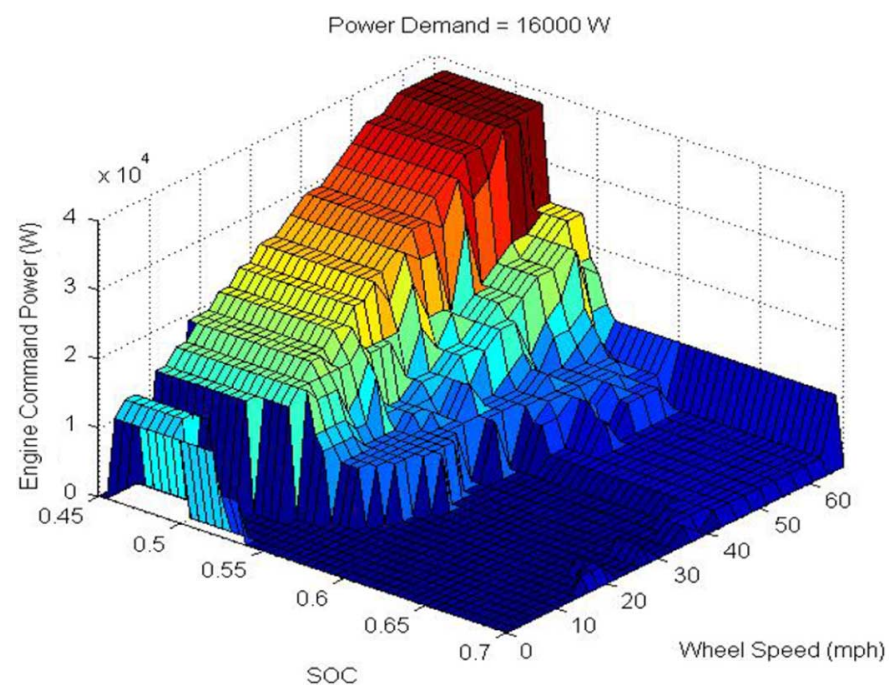

Fig. 8. Example of optimized engine power map from SDP.

necessary to assign a cost for the electric machine power. Otherwise, the optimization problem is not well posed. Given the fact that the battery SOC needs to be maintained at a proper level, $P_{\text {elec }}$ is not "free" and can be assigned an equivalent fuel consumption cost

$$
\dot{m}_{f_{-} \text {total }}=\dot{m}_{f_{- \text {eng }}}+\dot{m}_{f_{- \text {elec }}}
$$

where $\dot{m}_{f_{-} \text {eng }}$ represents the fuel consumption of engine and $\dot{m}_{f \_ \text {elec }}$ represents the equivalent fuel consumption of the electric machines, which can be calculated from

$$
\dot{m}_{f \_ \text {elec }}=\frac{\overline{F C} \cdot P_{\text {elec }}}{\overline{\eta_{\text {trans }}}} .
$$

In (18), $\overline{\mathrm{FC}}$ is the estimated engine fuel consumption conversion factor. The average efficiency of battery, inverter, and motor/generator are considered by: $\overline{\eta_{\text {trans }}}=\overline{\eta_{\text {batt }} \cdot \eta_{i} \cdot \eta_{m / g}}$. Using (17), an approximated equivalent fuel consumption is obtained. The benefit of using this single conversion factor is that the fuel consumption can be estimated regardless of the speed and torque of the engine and the motor/generator. The drawback is that its accuracy is questionable when the driving cycle changes.

Another major problem of (17) is that it does not include the battery SOC and electric machine into consideration. To achieve SOC regulation, a weighting factor $f$ (soc) (see Fig. 9) was suggested in [20]. It sets the target SOC at around 0.6 and weighs the SOC away from this target value such that the equilibrium $\mathrm{SOC}$ is attractive.

The original ECMS algorithm proposed in [20] does not consider kinematic constrains imposed by electric machines. Kinematic constraints are more important in power-split hybrids because of the CVT nature of the power-split device. For example, from (5), by using parameters for the THS system, if the desired engine power is $20 \mathrm{~kW}$, then the optimal engine speed is $\omega_{e_{-} d}=2333 \mathrm{r} / \mathrm{min}$ to achieve optimal efficiency. Due to the MG1 speed limit of $6500 \mathrm{r} / \mathrm{min}$, the vehicle speed must be higher than $12.6 \mathrm{mi} / \mathrm{h}$ for the optimal engine speed to be realizable (as 


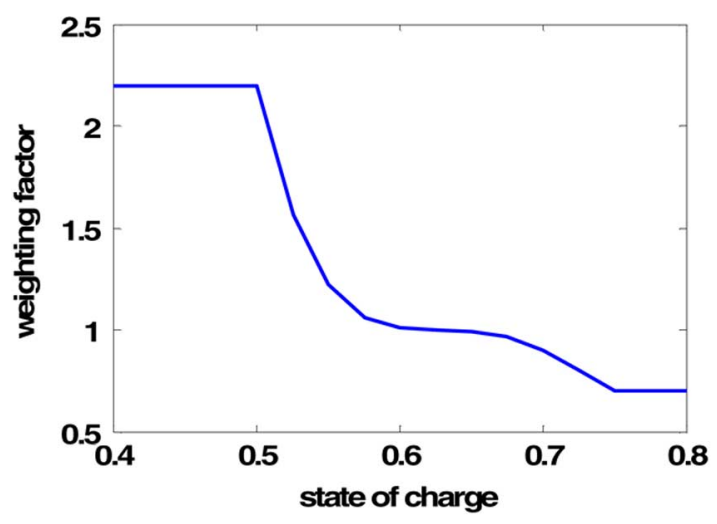

Fig. 9. SOC weighting factor $f$ (soc) for the ECMS algorithm [18].

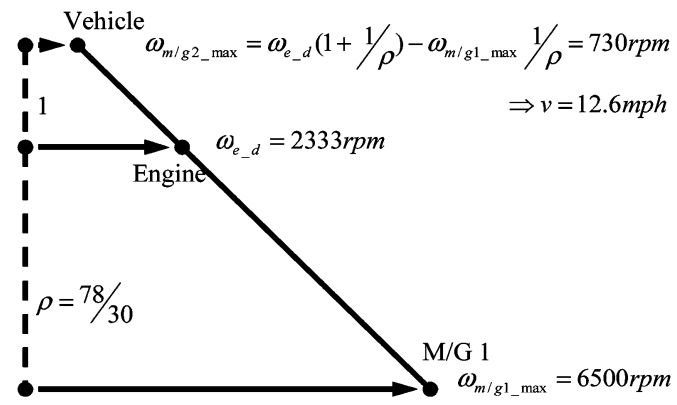

Fig. 10. Speed constraint calculation in THS.

shown in Fig. 10). At higher engine power demand, the optimal engine speed can be even higher, and the unreachable set imposed by the kinematic constraint grows even larger.

Due to the kinematic constraint, the feasible engine power is a function of power demand $P_{d}$ and vehicle speed $v$ [23]. In other words, (17) is modified to

$$
\dot{m}_{f_{-} \text {equi }}=\dot{m}_{f_{- \text {eng }}}\left(v, P_{d}\right)+f(\mathrm{soc}) \cdot \dot{m}_{f_{-} \text {batt }} .
$$

With this equivalent consumption cost function, given a power demand $P_{d}$, the optimal engine power can be searched among all feasible values to achieve minimal weighted equivalent fuel consumption. Fig. 11 shows the searching process for the condition with $P_{d}=30 \mathrm{~kW}, \mathrm{SOC}=0.6$, and $v=16 \mathrm{mi} / \mathrm{h}$. The fourth plot shows the combined equivalent fuel consumption without considering the kinematic constraints. However, with the kinematic constraints, the engine cannot operate in the shadowed region shown in the fifth plot, the optimal solution is hence on the boundary of the feasible region.

Repeat this process for all states, the calculated optimal engine power map is determined offline for each vehicle speed, one example is shown in Fig. 12.

\section{DYNAMIC PROGRAMMING ON THS}

To evaluate the two control approaches introduced in the previous sections, performance benchmarks will be obtained from the dynamic programming (DP) [24] technique. DP is a multi-stage decision-making process for dynamic systems. It generates optimal solution backwards along a time horizon by searching through all feasible discrete control actions for all the

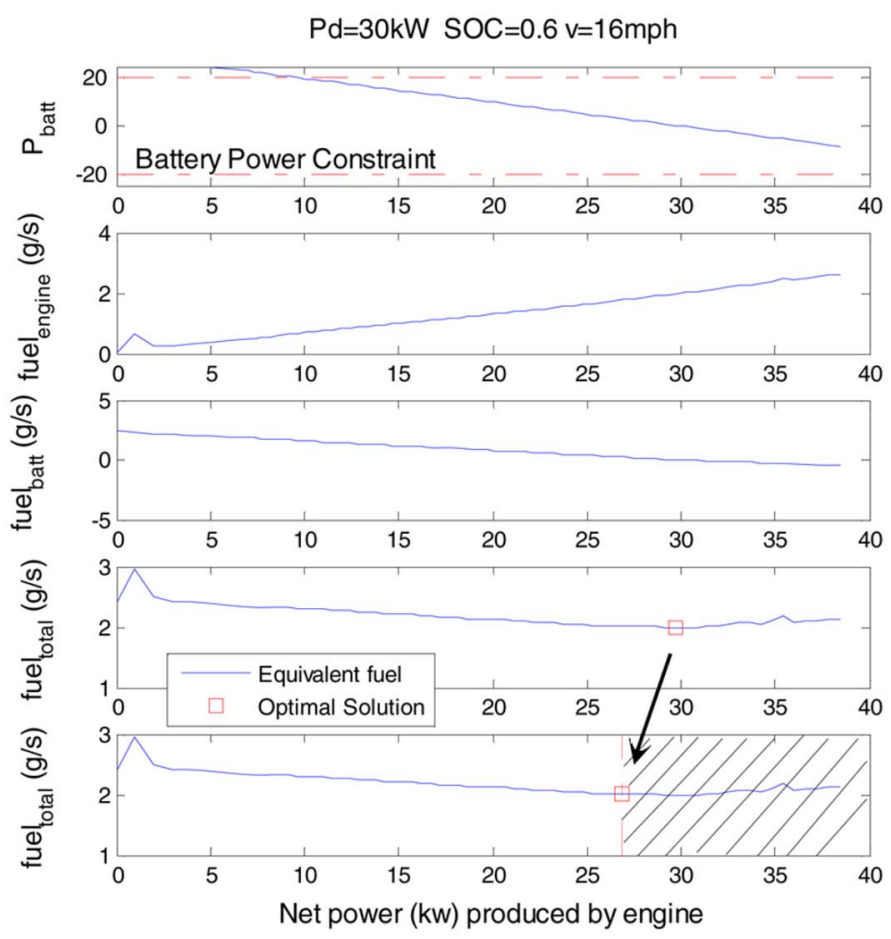

Fig. 11. Optimal solution searching process for the ECMS algorithm.

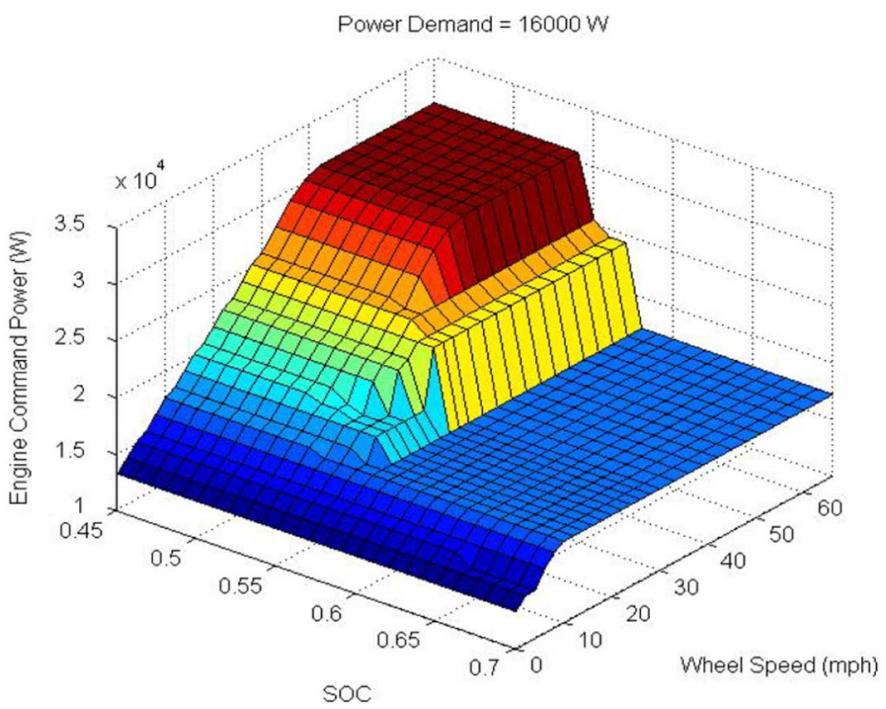

Fig. 12. Example optimized engine power map from ECMS.

state grid points. This algorithm guarantees global optimality up to the grid accuracy even under state and input constraints.

For the power-split hybrid vehicle studied in this paper, the DP algorithm searches solutions for two inputs (engine torque and MG1 torque), for two state variables: engine speed and battery SOC. The vehicle speed is not a free state variable to be searched because it is specified by the driving cycle. Similarly, if the cycle is to be followed by the power-train, only two input torques need to be specified-the MG2 torque is no longer free and can be calculated from

$$
T_{\mathrm{MG} 2}=\left(P_{d}-T_{e} \cdot \omega_{e}-T_{\mathrm{MG} 1} \cdot \omega_{\mathrm{MG} 1}\right) / \omega_{\mathrm{MG} 2} .
$$


TABLE II

SimUlation Time (EFFECT OF VeCTORIZATION APPROACH)

\begin{tabular}{ccc}
\hline \hline Simulation Cases & Simulation Approach & $\begin{array}{c}\text { Simulation } \\
\text { Time (sec) }\end{array}$ \\
$\begin{array}{c}\text { One engine speed } \\
\text { SOC grids [0.4:0.003:0.7] } \\
\begin{array}{c}\text { Throttle grids [0:0.025:1] } \\
\text { One MG1 Torque }\end{array}\end{array}$ & $\begin{array}{c}\text { Simulate one case at a } \\
\text { time }\end{array}$ & 153.6 \\
\hline \hline
\end{tabular}

The objective of the optimization problem is to minimize fuel consumption while following the driving demand and to keep the SOC within a reasonable range. The cost function is chosen to be

$$
\begin{aligned}
J & =\sum_{k=0}^{N-1}\left(\text { fuel }_{k}+\alpha \Delta_{\text {SOC }}^{2}\right) \\
\Delta_{\text {SOC }} & = \begin{cases}\mathrm{SOC}_{k}-\mathrm{SOC}_{d} & \mathrm{SOC}_{k}<\mathrm{SOC}_{d} \\
0 & \mathrm{SOC}_{k}>=\mathrm{SOC}_{d} .\end{cases}
\end{aligned}
$$

The optimization is also subject to the inequality constraints as shown in (14).

Because the DP search is exhaustive, it is computation intensive. In this study, several techniques are applied to reduce the computation load. First, the speed constraint can be examined without running the simulation. For example, knowing the vehicle speed and engine speed, MG1 speed can be calculated and if it violates the constraint, it can be assessed a large penalty and the simulation can be skipped. Second, SIMULINK allows us to load the simulation once and run a group of cases together. This is achieved through vectorizing the states and inputs. For instance, if the simulation model is loaded with an SOC vector [0.4:0.003:0.7], and an engine throttle vector [0:0.025:1], and specified scalar value of other states and inputs, SIMULINK actually takes in a matrix of cases, and all scenarios specified by the matrix can be simulated in one function call. Table II shows the comparison of computation time requirement between the two methods. It can be seen that the computation time is reduced by a factor of 300 . Third, to further accelerate simulations, the SIMULINK model can be converted into script (.m) file. When all the simulations for calculating transition table are made in an $\mathrm{m}$-file, without loading the SIMULINK model from the program, it further reduces the computation time by a factor of 10. With the help of all these techniques, the transition table computation for the UDDS driving cycle which took days was generated in about three hours on a desktop PC.

\section{RESULT AND DISCUSSION}

Simulations of the same vehicle model with SDP and ECMS controllers are conducted under various driving cycles to evaluate the control performances. The effect of mismatched SOC at the beginning and end of the cycle is compensated for by conducting several simulations with different initial values of SOC. As shown in Fig. 13, the fuel consumption changes monotonically and approximately linearly with the SOC difference. The fuel efficiency with zero SOC variation can then be calculated by interpolation.

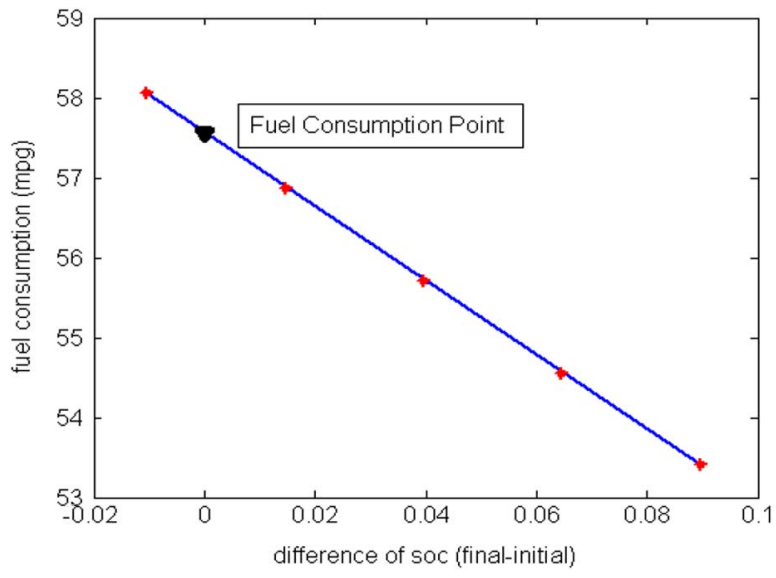

Fig. 13. Relationship between fuel consumption and change in battery.

TABLE III

FuEl ECONOMY COMPARISON BETWEEN DIFFERENT CONTROL ALGORITHMS

\begin{tabular}{ccccc}
\hline \hline $\begin{array}{c}\text { Fuel } \\
\begin{array}{c}\text { Consumption } \\
\text { (mpg) }\end{array}\end{array}$ & $\begin{array}{c}\text { Rule-Based } \\
\text { Control }\end{array}$ & $\begin{array}{c}\text { SDP } \\
\text { Control }\end{array}$ & $\begin{array}{c}\text { ECMS } \\
\text { Control }\end{array}$ & DP \\
UDDS & 57 & 65 & 64 & 67 \\
HWFET & 54 & 57 & 56 & 57 \\
\hline \hline
\end{tabular}

The SOC-corrected fuel economy results of the three control algorithms are presented in Table III. The results using the rule-based control algorithm, which is an approximation of the control law used in the commercial THS vehicle, are also reported for comparison. Both the SDP and ECMS algorithms show significant fuel economy improvement and both are close to the optimal results produced by DP. Results in Table III seem to validate that the SDP approach and the ECMS approach are near-optimal and are good candidates for developing control algorithms for implementation.

Improved overall fuel efficiency can be obtained only with improved engine efficiency. To examine the instantaneous engine performance, the engine operating points for both SDP and ECMS approaches are shown in the engine brake specific fuel consumption (BSFC) maps in Fig. 14. The total numbers of visitations are highlighted in different colors. The contours of equi-BSFC lines show the relative fuel efficiency of the operating points. In addition, the most efficient points for given engine power are shown by the red dashed line. Close examination of this figure confirms the engine operates very close to the theoretical optimum points, probably through utilization of the electric machines.

In spite of the similarities, the distributions of the engine operating points shown in Fig. 14 also have noticeable differences. The engine power traces, commanded by DP, SDP, and ECMS algorithms during a vehicle launch are plotted in Fig. 15. It can be seen that the engine power commanded by the ECMS oscillates continuously. This is partly due to the fact that the best engine efficiency is obtained with relatively high engine power (as shown in Fig. 14). When the power demand is low, the instantaneous optimization algorithm tends to move the engine toward a more efficient point, which generates more power than 

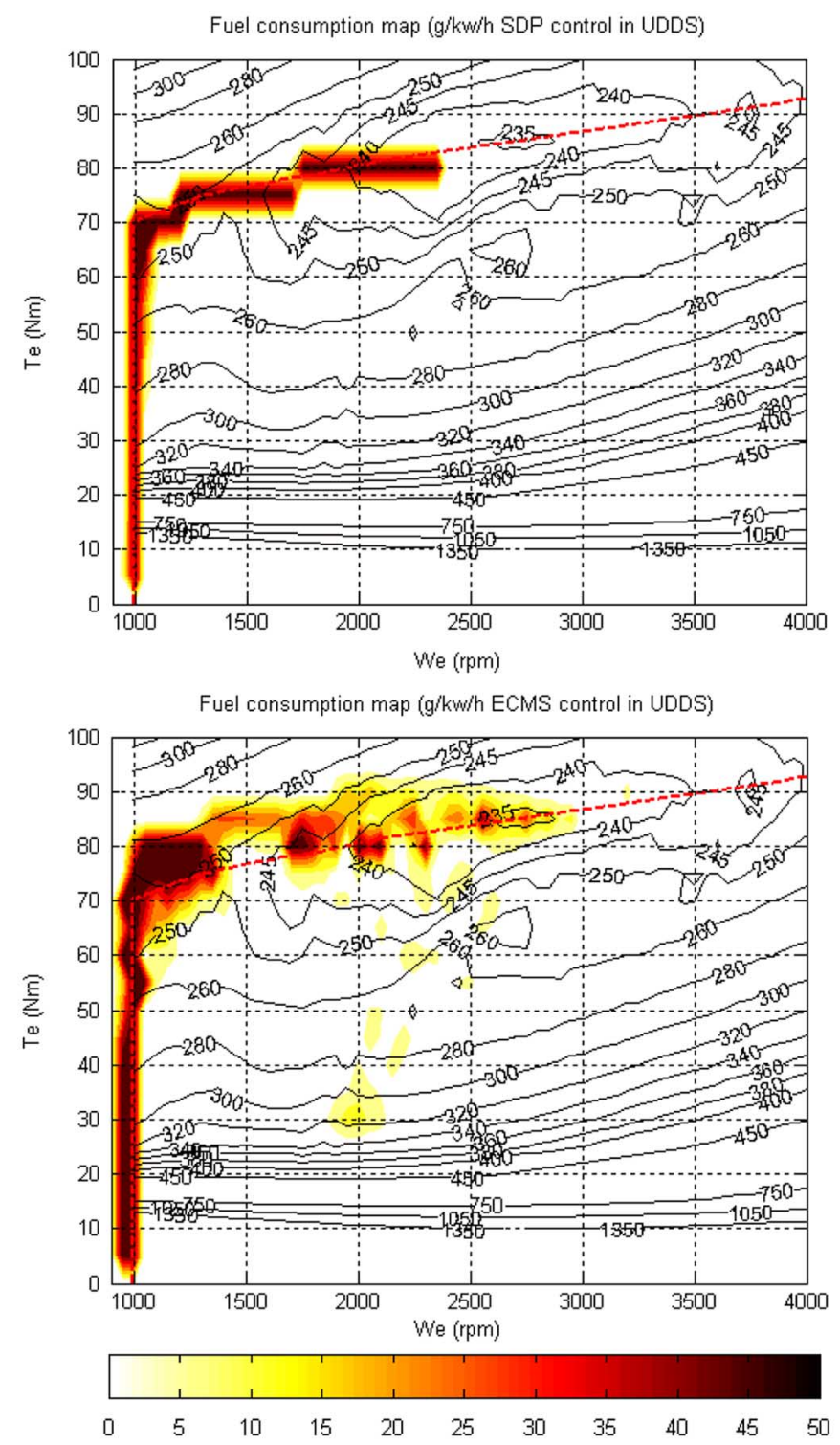

Fig. 14. Engine operating points densities for both SDP and ECMS approaches in UDDS cycle. (Sampling: $1 \mathrm{~Hz}$.)

demanded. The extra power delivered is balanced by the electrical path and the excessive energy is stored in the battery. This saved energy is then used to assist engine operation, allowing the engine to generate less power than the vehicle load required. This results in the widely varying engine power, which is also responsible for the scattered engine power generation shown in Fig. 14. The engine power generated by the SDP algorithm, in comparison, is much smoother. Since the SDP strategy is obtained based on infinite-horizon optimization, the future is taken into consideration, albeit in a stochastic way. Due to the longer optimization horizon, the SDP results do not react to instantaneous condition excessively.

The DP power flow presented in Fig. 15 is used to evaluate the power decisions made by the two control strategies. It shows an attempt similar to ECMS during the vehicle launch when the vehicle speed is low, but is much smoother for the rest of the sample cycle. The SDP approach produces smoother
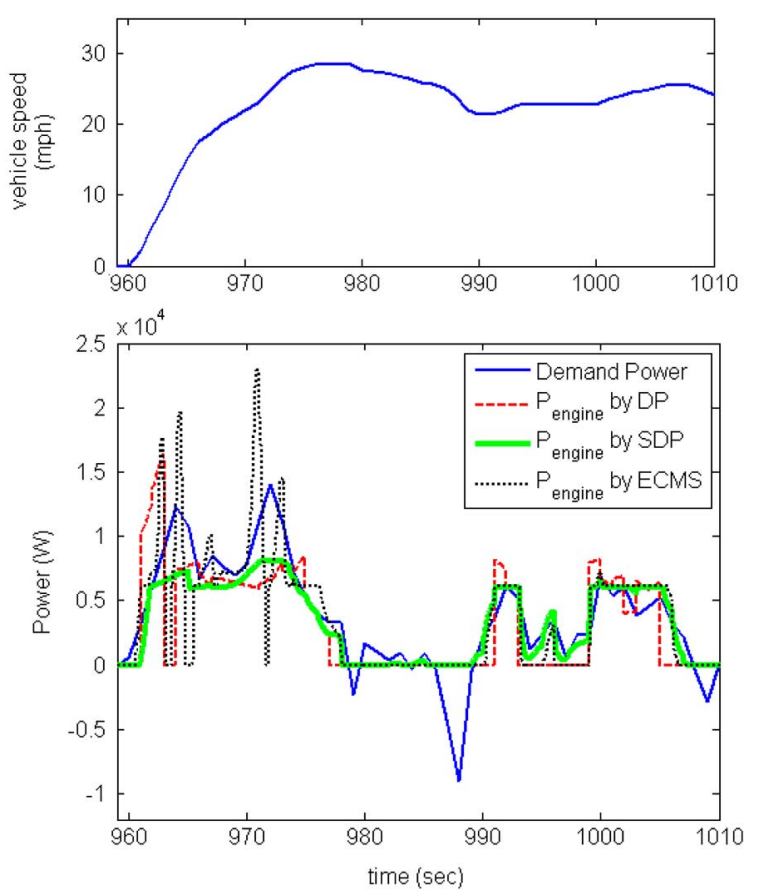

Fig. 15. Engine power by DP, SDP, and ECMS algorithms during a vehicle launch.

power compared with ECMS which is desirable from the drivability viewpoint. The fuel consumptions of these two algorithms, shown in Table III, however, are very similar.

The three control strategies discussed in this paper can be extended to study more complicated configurations with extended control design objectives. Emission, as an example, is another important concern for hybrid vehicle control. To add the emission constraints in the control, in DP or SDP, the cost function can be changed to have weighted emission associated terms. By tuning the weighting factors, the compromise between fuel consumption and emission can be achieved [25]. In the ECMS approach, equivalent fuel consumption cost regarding to the emission can be estimated to penalize the engine usage. Another popular power-split system under development is the dual-mode Alison Hybrid System from GM [26]. It utilized clutches to shift between different operating modes to achieve better efficiency. As for its optimal control design, DP or SDP need to add the operating gear mode as another input. The addition of another search dimension may result of heavy computational burden due to the "curse of dimension" from these approaches. In ECMS, the equivalent fuel consumption calculated in different modes can be compared to command the engine power. Frequent shifting should be prohibited by adding extra constraints between the gears.

\section{CONCLUSION}

A dynamic model for power-split hybrid vehicles is developed in this paper. This hybrid vehicle uses a single planetary gear and has a configuration similar to the THS. This model uses published mechanical and electric parameters from THS and THS II and its behavior was verified to be qualitatively similar to the experimental results based on Toyota Prius. 
Based on this dynamic model, two different control approaches are designed based on the SDP and ECMS. Both approaches determine the engine power requests based on the overall vehicle efficiency and apply the two electrical machines in the THS powertrain to augment the engine operation. The main difference lies in the fact the SDP approach assumes a stochastic driving power demand and the optimization is based on expected performance over an infinite horizon. In contrast, the ECMS approach uses a conversion factor to estimate the equivalent fuel consumption incurred when electric power is utilized. The total fuel consumption is then minimized based on instantaneous driving condition, with an additional ad hoc weighting function to ensure charge sustaining. Both strategies had been studied before for parallel and series hybrid vehicles and are modified for power-split hybrids in this paper. The kinematic constraint imposed by the planetary gear was found to be a major factor to be considered in the development of the new SDP and ECMS algorithms. Both control strategies were found to be near-optimum, demonstrating excellent fuel economy in standard driving cycles in comparison to the benchmark but non-causal results obtained through DP. The ECMS algorithm was found to produce more "jumpy" engine power command in its unmodified form-a simple rate limiting or filtering approach obviously can be used to address this issue. Both design methods achieve excellent fuel economy results and seem to be excellent candidates for developing control algorithms for implementations.

\section{REFERENCES}

[1] C. C. Chan, "The state of the art of electric and hybrid vehicles," Proc. IEEE, vol. 90, no. 2, pp. 247-275, Feb. 2002.

[2] G. H. Gelb, N. A. Richardson, T. C. Wang, and B. Berman, "An electromechanical transmission for hybrid vehicle power trains-design and dynamometer testing," SAE, Warrendale, PA, Tech. Rep. 710235, Jan. $11-15,1971$.

[3] J. M. Miller and M. Everett, "An assessment of ultra-capacitors as the power cache in toyota THS-II, GM-allision AHS-2 and ford FHS hybrid propulsion systems," in Proc. 20th Annu. Appl. Power Electron. Conf. Expo., 2005, vol. 1, pp. 481-490.

[4] S. Sasaki, "Toyota's newly developed hybrid powertrain," presented at the IEEE 10th Int. Symp. Power Semicond. Devices ICs, Kyoto, Japan, Jun. 1998.

[5] D. Hermance, "Toyota hybrid system," presented at the SAE TOPTEC Conf., Albany, NY, May 1999.

[6] A. Kimura, T. Abe, and S. Sasaki, "Drive force control of a parallelseries hybrid system," J. SAE Rev., vol. 20, pp. 337-341, 1999.

[7] S. Abe, "Development of the hybrid vehicle and its future expectation," SAE, Warrendale, PA, Tech. Rep. 2000-01-C042, 2000.

[8] M. Duoba, H. Ng, and R. Larsen, "In-situ mapping and analysis of the toyota prius HEV engine," SAE, Warrendale, PA, Tech. Rep. 2000-013096, 2000.

[9] A. Rousseau, P. Sharer, and M. Pasquier, "Validation process of a HEV system analysis model: PSAT," SAE, Warrendale, PA, Tech. Rep. 2001-01-0953, 2001

[10] Advanced Vehicle Simulator, Golden, CO, "National renewable energy laboratory," 2005 [Online]. Available: http://www.ctts.nrel.gov/analysis/advisor.html

[11] M. Duoba, H. Ng, and R. Larsen, "Characterization and Comparison of two hybrid electric vehicles (HEVs) - Honda insight and Toyota Prius," SAE, Warrendale, PA, Tech. Rep. 2001-01-1335, 2001.

[12] K. Muta, M. Yamazaki, and J. Tokieda, "Development of new-generation hybrid system THS II-Drastic improvement of power perfomance and fuel economy," SAE, Warrendale, PA, Tech. Rep. 2004-010064, 2004.
[13] T. Yaegashi, S. Abe, and D. Hermance, "Future automotive powertrain-Does hybridization enable ICE vehicles to strive towards sustainable development," SAE, Warrendale, PA, Tech. Rep. 2004-21-0082, 2004.

[14] A. Kawahashi, "A new-generation hybrid electric vehicle and its implications on power electronics," presented at the Power Electron. Seminar Ind. Rev. (CPES), Blacksburg, VA, Apr. 18-20, 2004.

[15] D. Hermance and S. Abe, "Hybrid vehicles lessons learned and future prospects," SAE, Warrendale, PA, Tech. Rep. 2006-21-0027, 2006.

[16] D. Rizoulis, J. Burl, and J. Beard, "Control strategies for a series-parallel hybrid electric vehicle," SAE, Warrendale, PA, Tech. Rep. 2001-01-1354, 2001.

[17] H. Zhang, Y. Zhu, G. Tian, Q. Chen, and Y. Chen, "Optimal energy management strategy for hybrid electric vehicles," SAE, Warrendale, PA, Tech. Rep. 2004-01-0576, 2004.

[18] J. M. Miller, "Hybrid electric vehicle propulsion system architectures of the E-CVT type," IEEE Trans. Power Electron., vol. 21, no. 3, pp. 756-767, May 2006.

[19] C. C. Lin, H. Peng, and J. W. Grizzle, "A stochastic control strategy for hybrid electric vehicles," presented at the Amer. Control Conf., Boston, MA, Jun. 2004

[20] G. Paganelli, Y. Guezennec, and G. Rizzoni, "Optimizing control strategy for hybrid fuel cell vehicle," SAE, Warrendale, PA, Tech. Rep. 2002-01-0102, 2002.

[21] H. Benford and M. Leising, "The lever analogy: A new tool in transmission analysis," SAE, Warrendale, PA, Tech. Rep. 810102, 1981.

[22] J. Liu, H. Peng, and Z. Filipi, "Modeling and control analysis of Toyota hybrid system," presented at the Int. Conf. Adv. Intell. Mechatron., Monterey, CA, Jul. 2005.

[23] J. Liu and H. Peng, "Control optimization for a power-split hybrid vehicle," presented at the Amer. Control Conf., Minnesota, MN, Jun. 2006.

[24] R. E. Bellman, Dynamic Programming. Princeton, NJ: Princeton Univ. Press, 1957.

[25] C. C. Lin, H. Peng, J. W. Grizzle, and J. Kang, "Power management strategy for a parallel hybrid electric truck," IEEE Trans. Control Syst. Technol., vol. 11, no. 6, pp. 839-849, Nov. 2003.

[26] B. Conlon, "Comparative analysis of single and combined hybrid electrically variable transmission operating modes," SAE, Warrendale, PA, Tech. Rep. 2005-01-1162, 2005.

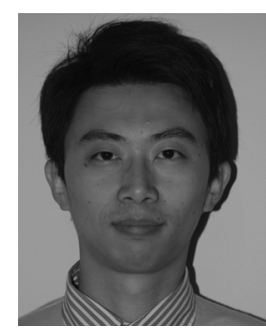

Jinming Liu received the $\mathrm{Ph} . \mathrm{D}$. degree in mechanical engineering from the University of Michigan, Ann Arbor, in 2007, the M.S. degree in manufacturing engineering from the University of Missouri, Rolla, in 2003, and the B.S. degree in mechanical engineering from Tsinghua University, Tsinghua, China, in 2001.

$\mathrm{He}$ is currently working with General Motors for Hybrid Architecture Engineering. His research interests include the areas of hybrid vehicle powertrain architecture design and controls, vehicle systems modeling and simulation, control system design, and

applications

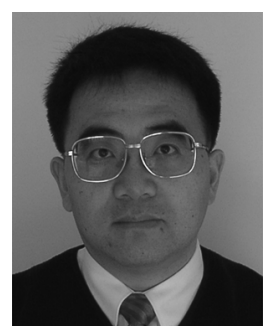

Huei Peng received the Ph.D. degree in mechanical engineering from the University of California, Berkeley, in 1992.

$\mathrm{He}$ is currently a Professor with the Department of Mechanical Engineering, University of Michigan, Ann Arbor. His research interests include adaptive control and optimal control, with an emphasis on their applications to vehicular and transportation systems.

Prof. Peng was a recipient of the National Science Foundation (NSF) Career Award in 1998. He has been an active member of SAE and the ASME Dynamic System and Control Division. He is currently an Associate Editor for the ASME Journal of Dynamic Systems, Measurement and Control. 